Conclusions This study documents the annual incidence of NSIs among HCWs showing important potential exposure to viral hepatitis and HIV, as well as the improvement in needlestick prevention over the years in Taiwan. In addition, the provision of safety engineered devices became a law in December 2011 in Taiwan. It is worth observing the influence of the new act to HCW's occupational health.

\section{BURNOUT SYNDROME AND COMMON MENTAL DISORDERS IN PEDIATRIC NURSING WORKERS}

C P Baptista, Tito, Silva, Vasconcelos, ea Felli. University of Sao Paulo, Sao Paulo, Brazil

\subsection{6/oemed-2013-101717.46}

Objectives Burnout syndrome is characterised by chronic stress related to work, in which there has been a gradual physical and mental strain process. The consequences to workers are related to their professional, familiar and social relationship. The nursing care in paediatric cardiology has been reported as very painful and distressing, and thus generating mental disorders. Aimed to identify the presence of burnout and common mental disorders (CMD) in nursing workers of a paediatric cardiology unit. Methods This study was conducted with 92 nursing workers of a paediatric cardiology unit of a publicBrazilianUniversityHospital. The Maslach Burnout Inventory (MBI), the Self Reporting Questionnaire (SRQ-20) and a questionnaire to characterise socio-demographic data were used to data collection. Data analysis considered chi-square test and Pearson correlation coefficient to associate the SRQ-20 and socio-demographic variables, and the SRQ-20 with the three dimensions assessed by the MIB.

Results The results showed that the nursing workers have on average 31 years old, $93.5 \%$ were female, $3 \%$ worked less than 10 years in a paediatric and neonatal cardiology unit, and $79.3 \%$ have only one employment. It was observed burnout in 8 nursing workers $(8.7 \%)$ and $41(45.0 \%)$ with CMD. Considering the socio-demographic variables, the chi-square test didn't show significant association with burnout and CMD. However, the chisquare test showed significant association between burnout e CMD ( $\mathrm{p}<0.005)$. The Pearson correlation coefficient showed an correlation between CMD and the three dimensions of burnout, Emotional Exaustion ( $r=0.59)$, despersonalisation $(r=$ 0.44) and Reduce Personal Accomplishment ( $\mathrm{r}=-0.31$ ), a reverse score.

Conclusions This study showed association between the burnout and CMD and correlation with the three dimensions of burnout with the CMD. Future research should focus upon identifying paediatric nurses with CMD earlier, avoiding the burnout syndrome and abandonment of profession.

\section{PRELIMINARY STUDY OF OCCUPATIONAL STRESS IN A MOROCCAN HOSPITAL}

${ }^{1} \mathrm{~F}$ Z Azzaoui, ${ }^{2} \mathrm{H}$ Chtibi, ${ }^{3}$ Ahami, ${ }^{4} \mathrm{Hami} .{ }^{1}$ Faculty of Science, Kenitra, Morocco; ${ }^{2}$ IBN TOFAIL University, Kenitra, Morocco; ${ }^{3}$ Equip of Clinic and Cognitive Neurosciences and Health, Department of Biology, Kenitra, Morocco; ${ }^{4}$ Laboratory of Genetics and Biometry, Department of Biology, Kenitra, Morocco

\subsection{6/oemed-2013-101717.47}

Background Occupational stress has an impact on health of medical and paramedical staff, on their productivity and on their relationship with the patients.
Aim The aim of this study is to evaluate stress among medical and paramedical staff and compare the degree of this parameter between these groups.

Methods The study is realised in hospital in Rabat, Morocco, among 20 doctors and 54 paramedical staff. The test used is a numeric test which consists on capacity of person to join 11 points for drawing 2 squares and 1 triangle in every 15 subtests. Every subtest remains 40 seconds; the totality of the test lasts 600 seconds. The tested person must put headphones linked to the laptop, and hears during the test different noises. The degree of this noise pollution increases from a subtest to another. Number of write response during each subtest and the total scores are calculated.

Results The preliminary results show that the mean score of write responses obtained under noises among doctors are 47/90, among nurses in anaesthesia and resuscitation are 44/90, among nurses of laboratory are 43/90, among polyvalent nurses are $32 /$ 90 and among radiology nurses are 30/90. These scores are significantly different between doctors and all specialties of nurses $(\mathrm{p}<0.01)$. Moreover, the most affected groups by stress are polyvalent and radiology nurses compared to doctors $(\mathrm{p}<0.01)$ and compared to nurses in anaesthesia and resuscitation $(\mathrm{p}<$ $0.05)$, and to nurse of laboratory ( $<<0.05$ ).

Conclusion Nurses are more affected by occupational stress than doctors, especially polyvalent and radiology nurses. Several factors could be involved in this occupational pathology. So, deeper investigations are needed.

\section{HEALTH INDICATORS OF HEALTH WORKER IN A UNIVERSITY HOSPITAL: PRELIMINARY DATA}

${ }^{1}$ L L S Santana, ${ }^{2}$ da Cruz, ${ }^{2}$ Sarquis, 'Kalinke, ${ }^{3}$ Kirchhof, ${ }^{4}$ E A Felli. ' CURITIBA, Brazili; ${ }^{2}$ Universidade Federal do Paraná, CURITIBA, Brazil; ${ }^{3}$ Universidade Federal de Santa Catarina, Florianópolis, Brazil; ${ }^{4}$ Universidade de São Paulo, São Paulo, Brazil

\subsection{6/oemed-2013-101717.48}

Introduction Partial results of a dissertation of the Graduate Program in Nursing, Federal University of Parana - Brazil.

Objective To analyse the health indicators of a hospital worker in the year 2011.

Methods Retrospective epidemiological study conducted at a university hospital in southern Brazil whose data was collected through the database Monitoring System of Occupational Health Nursing (SIMOSTE) which consists of a technological tool developed by nursing researchers, with support from the Foundation to support research in São Paulo, aimed at monitoring the health of workers in a hospital environment.

Results Women $(80.8 \%)$ and workers aged 31 to 40 years $(34.2 \%)$ had more records of absences from work. The total absence corresponded to 2478 days, and 1526 (61.6\%) relative to the nursing staff. The highest average absence was observed among nursing technicians $(\mathrm{M}=2.63$ days $)$ and work sector with the highest proportion of registers was the Intensive Care Unit (23.2\%). The workload was the biological prevalent $(39.4 \%)$ and is also the main cause of attrition reported for 15 of the 23 occupational categories. Diseases of the respiratory system were the most away (19.62\%) and also in higher frequency registries (19.62\%). The highest number of days of sick leave (360 days) was due to diseases of the musculoskeletal system and connective tissue and the damage resulting from mental and behavioural disorders on average 5.76 days away. Nurses had 33 days of leave for respiratory diseases and an average of 4.71 days away from external causes. The nursing staff had more 